13

\title{
Исследование процессов зарядки ионно-имплантированных диэлектриков под воздействием электронного облучения
}

\author{
(С) Э.И. Рау, ${ }^{1,2}$ А.А. Татаринцев, ${ }^{1}$ Е.Ю. Зыкова, ${ }^{1}$ C.В. Зайцев ${ }^{1}$ \\ ${ }^{1}$ Московский государственный университет им. М.В. Ломоносова, \\ 119992 Москва, Россия \\ ${ }^{2}$ Институт проблем технологий микроэлектроники РАН, \\ 119117 Черноголовка, Россия \\ e-mail: rau@phys.msu.ru
}

Поступилов Редакцию 5 июля 2018 г.

В окончательной редакции 5 июля 2018 г.

Принято к публикации 20 февраля 2019 г.

Исследована кинетика зарядки диэлектриков $\mathrm{Al}_{2} \mathrm{O}_{3}$ (сапфира) и $\mathrm{SiO}_{2}$ ( $\alpha$-кварца), подвергнутых предварительному облучению ионами инертного газа $\mathrm{Ar}^{+}$, металла $\mathrm{Ga}^{+}$и протонами $\mathrm{H}^{+}$. Обнаружена существенная разница в кинетике зарядки образцов в зависимости от природы облучающих ионов. Установлено, что предварительное воздействие на диэлектрическую мишень ионизирующего корпускулярного излучения (протонов, ионов) значительно изменяет зарядовые характеристики поверхности диэлектриков. Эти различия зависят также от энергии облучающих ионов, определяющих глубину аккумулируемого слоя отрицательного заряда в сравнении с глубиной предварительной имплантации ионов.

Ключевые слова: электронная зарядка диэлектриков, ионное облучение, радиационные дефекты.

DOI: 10.21883/JTF.2019.08.47904.264-18

\section{Введение}

Известно, что облучение диэлектрических мишеней различными заряженными частицами, такими как электроны, протоны и ионы, может приводить к зарядке их поверхности. В широко распространенных в настоящее время методах диагностики поверхности электронными и ионными пучками зарядка диэлектрических поверхностей является негативным явлением, затрудняющим получение количественных данных. Большой проблемой является также электрическая зарядка корпусов космических аппаратов, представляющая угрозу их нормальному функционированию.

Несмотря на то, что изучение явления зарядки под воздействием электронного облучения ведется уже длительное время, в этой области остается еще много неясных и спорных моментов. В частности, мало изучен процесс зарядки диэлектрических мишеней с предварительно модифицированной поверхностью, например, диэлектриков, легированных различными примесями или подвергнутых радиационному воздействию. Способность диэлектриков накапливать заряд во многом зависит от наличия в приповерхностной области мишени структурных дефектов, которые являются ловушками для носителей заряда - электронов и дырок. По этой причине, например, поверхность аморфных диэлектриков, содержащая больше дефектов, заряжается более интенсивно по сравнению с поверхностью монокристаллов. В работе [1] было отмечено увеличение зарядовой способности легированного атомами металлов диэлектрика $\mathrm{Al}_{2} \mathrm{O}_{3}$ при его облучении электронами в сканирующем электронном микроскопе (СЭМ).
При ионной бомбардировке поверхности твердых тел происходит целый ряд различных процессов как упругих (распыление, образование дефектов, имплантация ионов), приводящих к изменению состава поверхности и ее кристаллической структуры, так и неупругих (перезарядка ионов, ионно-электронная эмиссия), приводящих к зарядки образца [2]. При бомбардировке диэлектрического образца положительными ионами имплантированные атомы создают под поверхностью внутреннее встроенное электрическое поле $F_{\text {in }}$, исходно поляризующее приповерхностный слой, причем вектор поляризации направлен от максимума плотности распределения ионов к поверхности. Вторым следствием ионной имплантации является генерация дополнительных ловушечных центров для свободных зарядов в области облучения. И, наконец, третье последствие ионного облучения диэлектрических мишеней - модификация состава и микрорельефа поверхности. Последний процесс зависит от дозы облучения, поэтому чтобы исключить образование микрошероховатостей на поверхности в наших экспериментах дозы $D_{0}^{+}$облучения ионами были относительно невелики $\left(D_{0}^{+}<10^{14} \mathrm{~cm}^{-2}\right)$. Модификация состава поверхности может приводить к изменению поверхностной электрической проводимости, и тем самым перераспределению плотности носителей заряда, что сильно сказывается на кинетике зарядки диэлектрических мишеней при экспериментах с электронным облучением в СЭМ. Все указанные факторы учтены в настоящей работе при интерпретации результатов исследований зарядки диэлектриков при их облучении электронами средних энергий. 
Таблица 1. Основные характеристики исследованных диэлектриков

\begin{tabular}{l|c|c}
\hline Характеристики & Сапфир $\left(\mathrm{Al}_{2} \mathrm{O}_{3}\right)$ & $\alpha$-кварц $\left(\mathrm{SiO}_{2}\right)$ \\
\hline $\begin{array}{l}\text { Диэлектрическая } \\
\text { постоянная } \varepsilon_{r}\end{array}$ & 9.5 & 3.9 \\
$\begin{array}{l}\text { Удельное } \\
\text { сопротивление } \rho, \Omega \cdot \mathrm{cm}\end{array}$ & $10^{12}$ & $10^{11}$ \\
$\begin{array}{l}\text { Ширина запрещенной } \\
\text { зоны } E_{g}, \mathrm{eV}\end{array}$ & 9 & 9 \\
$\begin{array}{l}\text { Максимальный коэффициент } \\
\text { эмиссии электронов } \sigma_{m} \\
\text { при энергии } E_{m}, \mathrm{keV}\end{array}$ & 6.2 & 3.6 \\
& 0.65 & 0.55
\end{tabular}

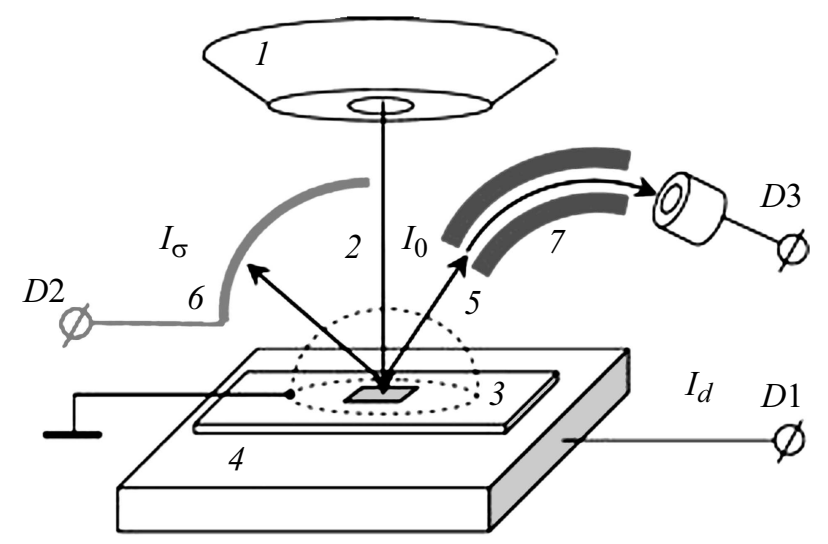

Рис. 1. Схема экспериментальной установки: 1 - объективная линза СЭМ, 2 - электронный пучок, 3 - диэлектрический образец, 4 - проводящая подложка, 5 - заземленная металлическая сетка, 6 - полусферический детектор электронов, 7 - тороидальный электронный энергоанализаторспектрометр, $D 1, D 2, D 3$ - детекторы сигналов.

\section{Экспериментальные методики и образцы для исследований}

В настоящей работе исследовались эмиссионные и зарядовые характеристики двух классических диэлектриков: монокристаллов сапфира $\left(\mathrm{Al}_{2} \mathrm{O}_{3}\right)$ и $\alpha$-кварца $\left(\mathrm{SiO}_{2}\right)$ при их электронном облучении. Образцы представляли собой пластины размером $10 \times 10 \times 0.5 \mathrm{~mm}$. Основные характеристики исследованных диэлектриков приведены в табл. 1, а в табл. 2 даны параметры предварительного ионного облучения. Основным требованием к экспериментальной установке для изучения процесса зарядки диэлектриков под электронным пучком было осуществление возможности одновременного измерения трех фундаментальных кинетических характеристик: коэффициента эмиссии электронов $\sigma(t)$, аккумулируемого в объеме диэлектрика заряда $Q T(t)$ и генерируемого в процессе облучения поверхностного потенциала $V_{s}(t)$. Именно синхронное одновременное измерение трех временных характеристик позволяет корректно интерпретировать сложные взаимозависимые и самосогласу- ющиеся процессы зарядки диэлектрических мишеней. Схема экспериментальной установки, инсталлированной в СЭM LEO-1455, приведена на рис. 1. Сформированный объективной линзой 1 электронный зонд 2 сканирует на исследуемой диэлектрической мишени 3 площадку $a \times a=100 \times 100 \mu \mathrm{m}^{2}$ в телевизионном формате разверток. Образец располагается на металлической подложке 4 , с которой снимается результирующий ток смещения и утечки $I_{d}$. Ток вторичных электронов $I_{\sigma}$ коллектируется усеченным полусферическим детектором 6 , покрытым изнутри антидинатронной угольной сажей. Поверхностные потенциалы $V_{s}$ регистрируются с помощью тороидального электронного энергоанализатора 7 методом детектирования сдвига спектров вторичных электронов по энергии.

Ток первичных электронов $I_{0}$ измеряется цилиндром Фарадея, расположенным рядом с образцом (на рис. 1 не показан). Для уменьшения влияния электростатических полей рассеяния на траектории ВЭ в пространстве над поверхностью образца помещена заземленная полусферическая металлическая сетка 5 с высокой оптической прозрачностью. Токовыми детекторами $D 1$ и $D 2$ являются пикоамперметры „Keithely“, а детектором $D 3$ спектрометра - микроканальная пластина „Hamamatsu“.

Для исследования влияния радиационного воздействия на процесс электронной зарядки диэлектрики были предварительно подвергнуты облучению ионами инертного газа $-\mathrm{Ar}^{+}$, ионами металла $\mathrm{Ga}^{+}$и протонами $\mathrm{H}^{+}$. Результаты зарядки этих образцов, предварительно облученных электронами, были отражены в наших предыдущих работах $[3,4]$.

\section{Результаты и их обсуждение}

Экспериментальные результаты исследования кинетики зарядки диэлектриков $\mathrm{Al}_{2} \mathrm{O}_{3}$ и $\mathrm{SiO}_{2}$, подвергнутых предварительному ионному облучению, приведены соответственно на рис. 2 и 3 . На рис. 2 представлены зависимости поверхностного потенциала монокристалла сапфира от времени облучения электронным пучком с плотностью тока $j_{0}=10^{-7} \mathrm{~A} / \mathrm{cm}^{2}$ и энергией $E_{0}=5 \mathrm{keV}(a)$ и $15 \mathrm{keV}(b)$. Аналогичные зависимости для $\mathrm{SiO}_{2}$ показаны на рис. 3 .

В первую очередь следует отметить различную кинетику зарядки исходных (необлученных) образцов сапфира и кварца (кривая 1 на рис. 2 и 3). Исходный образец сапфира практически не заряжается вплоть до времени $500 \mathrm{~s}$, затем потенциал поверхности начинает медленно увеличиваться и после $1000 \mathrm{~s}$ более быстро возрастает (рис. 2). В то же время поверхностный потенциал необлученной поверхности кварца мгновенно возрастает до равновесного значения $2.7 \mathrm{kV}$.

Такое различие в зарядке сапфира и кварца впервые было обнаружено нами в [3]. Это объясняется тем, что исследованный монокристалл кварца изначально имеет достаточно большое количество структурных дефектов, 
Таблица 2. Параметры предварительного протонного и ионного излучения сапфира и кварца

\begin{tabular}{c|c|c|c|c|c|c}
\hline & \multirow{2}{*}{ Элемент } & $\begin{array}{c}\text { Энергия, } \\
\mathrm{keV}\end{array}$ & $\begin{array}{c}\text { Плотность } \\
\text { ионного } \\
\text { тока, } \mathrm{A} / \mathrm{cm}^{2}\end{array}$ & $\begin{array}{c}\text { Доза } \\
\mathrm{cm}^{-2}\end{array}$ & \multirow{2}{*}{$\begin{array}{c}\text { Размер } \\
\text { области } \\
\text { облучения }\end{array}$} & \multicolumn{2}{|c}{$\begin{array}{c}\text { Глубина } \\
\text { имплантации, } \mathrm{nm}\end{array}$} \\
\cline { 5 - 8 } & 10 & $10^{-4}$ & $10^{17}$ & $3 \mathrm{~mm}$ & 8.2 & 13.9 \\
\hline $\mathrm{Ar}^{+}$ & 15 & $10^{-5}$ & $8 \cdot 10^{16}$ & $500 \times 500 \mu \mathrm{m}$ & 9 & 15.3 \\
\hline $\mathrm{Ga}^{+}$ & 50 & & $10^{14}$ & $3 \mathrm{~mm}$ & 319.3 & 515.4 \\
\hline $\mathrm{H}^{+}$ & 150 & & $10^{14}$ & $3 \mathrm{~mm}$ & 801.9 & 1302
\end{tabular}
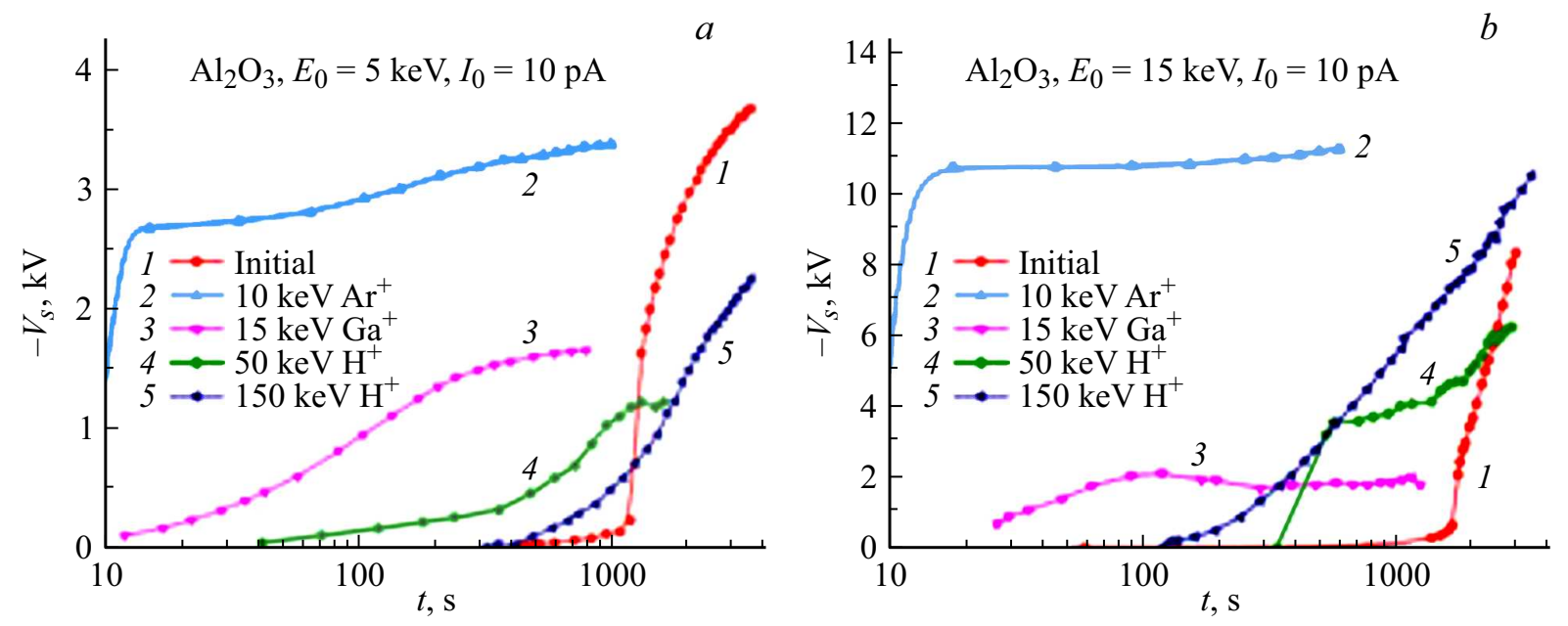

Рис. 2. Зависимость поверхностного потенциала сапфира от времени облучения электронным пучком с плотностью тока $j_{0}=10^{-7} \mathrm{~A} / \mathrm{cm}^{2}$ и с энергией $E_{0}=5 \mathrm{keV}(a)$ и $E_{0}=15 \mathrm{keV}(b)$. Кривая 1 потенциал зарядки исходной поверхности, $2-$ после облучения ионами $\mathrm{Ar}^{+}$с энергией $E_{0}=10 \mathrm{keV}, 3-$ после облучения ионами $\mathrm{Ga}^{+}$с энергией $15 \mathrm{keV} ; 4-$ после облучения протонами с энергией $50 \mathrm{keV} ; 5$ - после облучения протонами с энергией $150 \mathrm{keV}$.

с которыми ассоциированы электронные уровни, лежащие внутри запрещенной зоны кристалла. Эти уровни могут служить ловушками для первичных электронов, и поэтому поверхность монокристалла кварца заряжается относительно быстро, захватывая электроны на уже существующие ловушки. Это предположение отчасти подтверждается тем, что скорость зарядки $\mathrm{SiO}_{2}$ после предварительного облучения ионами $\mathrm{Ar}^{+}$и $\mathrm{Ga}^{+}$(кривые 2 и 3 соответственно на рис. 3) лишь немного превышает аналогичную скорость для исходного образца. Это означает, что дополнительные радиационные дефекты, созданные ионным облучением, оказывают меньшее влияние на процесс зарядки кварца под электронным пучком, чем для сапфира (кривые 2 и 3 на рис. 2).

$\mathrm{B}$ отличие от $\mathrm{SiO}_{2}$ исходный сапфир практически не имеет дефектов кристаллической структуры, поэтому поверхностный потенциал начинает расти только после того, как в кристалле под воздействием электронного пучка создаются радиационно-стимулированные дефекты, в частности вакансии кислорода, которые являются ловушками для электронов (кривая 1 на рис. 2).
Кроме того, в случае сапфира наблюдается резкое увеличение скорости зарядки после предварительного облучения ионами $\mathrm{Ar}^{+}$(кривые 2 на рис. 2), которое объясняется интенсивным захватом электронов на дефекты, генерированные предварительным ионным облучением в тонком приповерхностном слое. К числу таких дефектов относятся кислородные вакансии $F$ - и $F^{+}$-центры, электронные уровни которых лежат соответственно на 3 и $3.8 \mathrm{eV}$ ниже дна зоны проводимости [5], т. е. являются глубокими ловушками для электронов.

Следует отметить, что кинетика зарядки поверхности сапфира и достигаемый равновесный потенциал различаются в случае предварительного облучения ионами инертного газа $\mathrm{Ar}^{+}$и ионами металла $\mathrm{Ga}^{+}$. После предварительного облучения ионами $\mathrm{Ar}^{+}$(кривая 2) сапфир практически мгновенно заряжается до высокого значения поверхностного потенциала $V_{s}=2.7 \mathrm{kV}$, затем потенциал медленно нарастает, выходя на равновесное значение $V_{s}^{\infty}=3 \mathrm{kV}$. Скорость зарядки образца после облучения ионами $\mathrm{Ga}^{+}$(кривая 3) несколько ниже, а равновесное значение поверхностного потенциала $V_{s}^{\infty}$ составляет $\sim 1.6 \mathrm{kV}$, что значительно ниже, чем для 
$a$

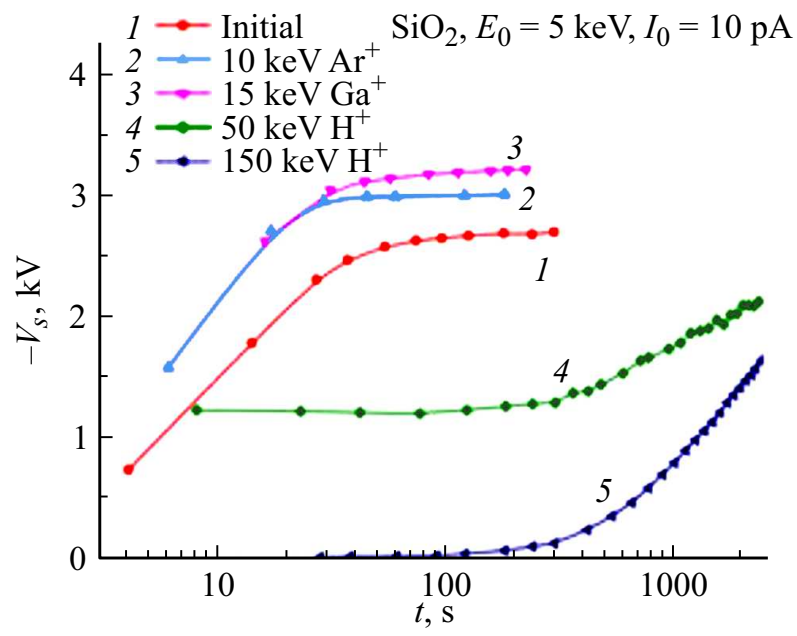

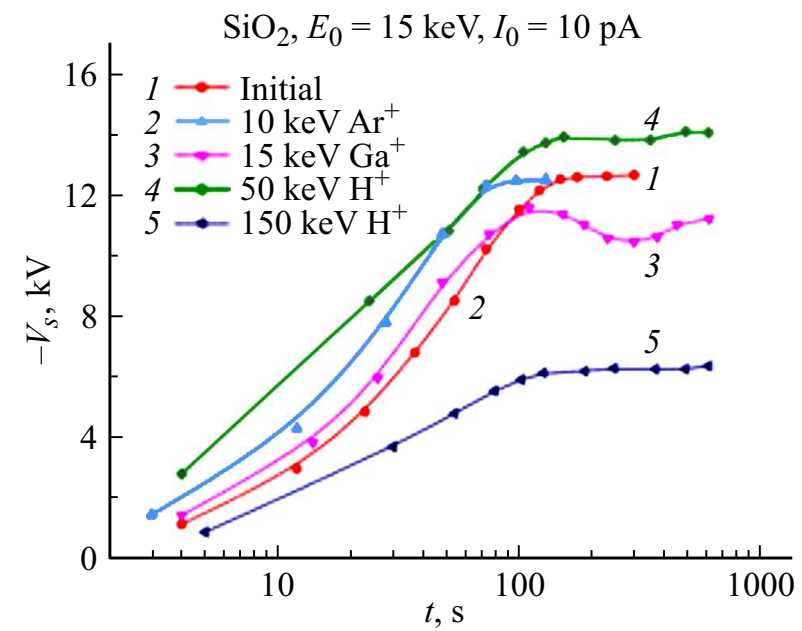

Рис. 3. То же, что и на рис. 2, но для кварца.

случаев необлученной поверхности и поверхности, предварительно облученной электронами или ионами $\mathrm{Ar}^{+}$.

Полученный результат указывает на меньшее количество глубоких электронных ловушек, оказывающихся в кристалле сапфира после его облучения ионами галлия, что может быть объяснено химическим эффектом. В работах [6,7] было установлено, что химическая природа ионов играет существенную роль во взаимодействии ионов с веществом. В частности, было обнаружено, что в случае имплантации ионов $\mathrm{Hg}^{+}$аморфизация поверхности сапфира происходит при дозах, на порядок более низких по сравнению с имплантацией ионами $\mathrm{Pt}^{+}$. Кроме того, было показано, что часть имплантированных ионов $\mathrm{Hg}^{+}$связывается в решетке с кислородом, образуя кластеры $\mathrm{HgO}$, расположенные в междоузлиях кристаллической решетки. В то же время 70\% ионов $\mathrm{Pt}^{+}$занимают регулярные позиции в подрешетке Al. Образование кластеров $\mathrm{ZnO}$ при имплантации ионов $\mathrm{Zn}^{+}$в сапфир было обнаружено в работе [8], $\mathrm{GaO}$ при имплантации в $\mathrm{SiO}_{2}$ ионов $\mathrm{Ga}^{+}$- в работе [9]. По аналогии можно предположить, что в наших экспериментах в результате облучения сапфира металлическими ионами $\mathrm{Ga}^{+}$часть имплантированных атомов $\mathrm{Ga}$ образуют в кристалле химическую связь с атомами кислорода, создавая в решетке кластеры $\mathrm{GaO}$. В результате количество междоузельных атомов кислорода уменьшается, что приводит к уменьшению количества глубоких электронных ловушек в приповерхностной области кристалла. Так что зарядка поверхности происходит медленнее, и равновесный отрицательный потенциал достигает меньшего значения.

На процесс зарядки диэлектриков под электронным пучком также может влиять положительная зарядка поверхности, возникающая в результате ионной имплантации. Полная картина механизмов зарядки под действием ионного облучения до конца еще не ясна, поэтому ограничимся кратким изложением процессов, проис- ходящих при имплантации ионов в диэлектрики [9]. При предварительном ионном облучении падающие высокоэнергетичные ионы по мере продвижения вглубь образца теряют свою энергию на генерацию электроннодырочных пар. Непосредственно перед остановкой в кристалле ион создает большое количество точечных дефектов в анионной и катионной подрешетках, которые служат глубокими или мелкими ловушками для электронов и дырок. В результате после остановки ион нейтрализуется, захватывая возбужденный свободный электрон, а оставшаяся дырка локализуется на глубоком уровне дефекта. Поверхность образца при этом заряжается до некоторого положительного потенциала, причем заряд аккумулируется в узкой приповерхностной области, поскольку глубина проникновения ионов $\mathrm{Ga}^{+}$ и $\mathrm{Ar}^{+}$с энергиями 10-15 keV в сапфире и кварце не превышает $20 \mathrm{~nm}$. Таким образом, снижение скорости электронной зарядки в случае предварительного облучения ионами $\mathrm{Ga}^{+}$может быть связано с необходимостью компенсировать больший положительный потенциал, чем в случае облучения ионами $\mathrm{Ar}^{+}$.

На рис. 2 и 3 приведены также кривые кинетики зарядки образцов после их предварительного облучения протонами (кривые 4 и 5). Видно, что в случае энергии электронов в СЭМ $E_{0}=5 \mathrm{keV}$ скорость нарастания поверхностного потенциала облученного протонами сапфира намного меньше, чем после облучения ионами $\mathrm{Ga}^{+}$ и тем более $\mathrm{Ar}^{+}$. Темп зарядки кварца также резко замедляется после его облучения протонами. В случае энергии электронов $E_{0}=15 \mathrm{keV}$ такой однозначности нет. Например, кварц, предварительно облученный протонами с энергией $E_{\mathrm{H}^{+}}=50 \mathrm{keV}$, заряжается быстрее и до более высокого равновесного потенциала.

Изменение со временем потенциала поверхности измерялось в зависимости от энергии протонов $E_{\mathrm{H}^{+}}=50$ и $150 \mathrm{keV}$, а также в зависимости от параметров последующего электронного облучения плотности электронного 


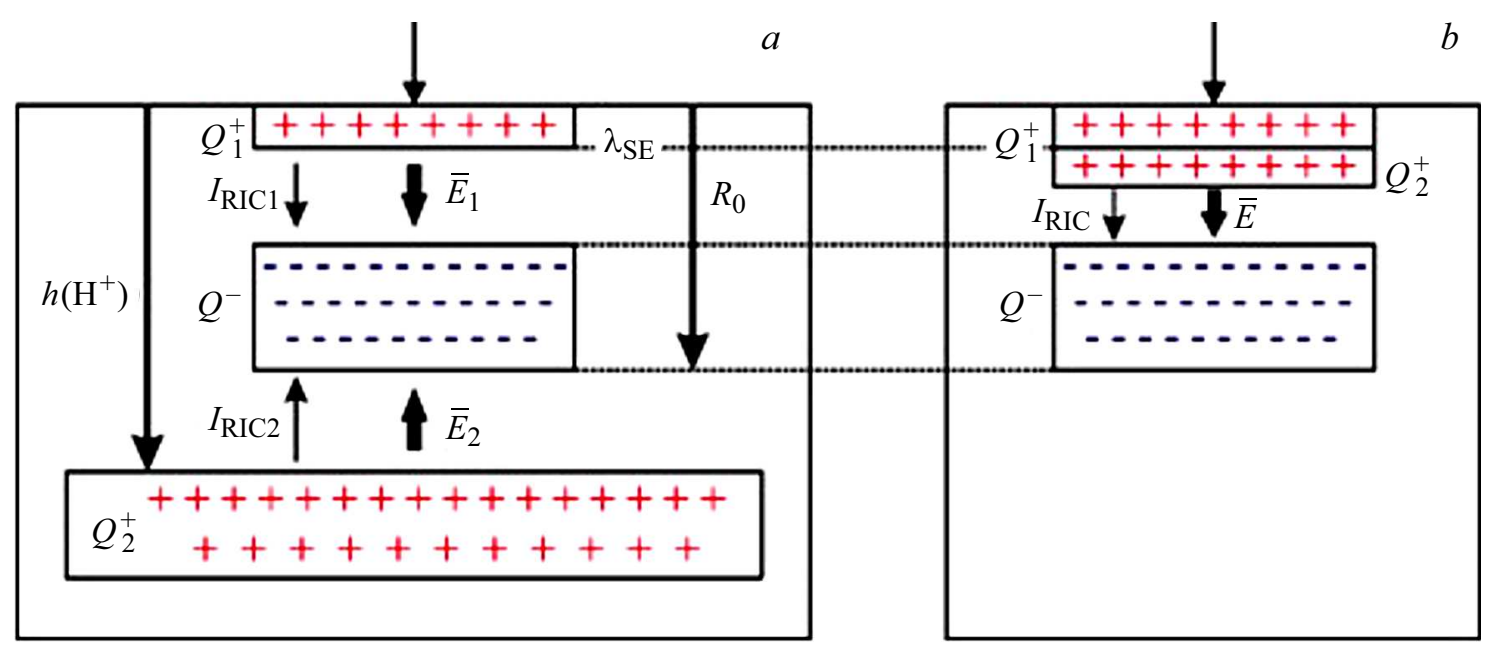

Рис. 4. Схема зарядки диэлектрического образца под электронным пучком: $a-$ зарядка после облучения протонами; $b-$ зарядка после ионного облучения.

тока $j_{0}=10^{-7} \mathrm{~A} / \mathrm{cm}^{2}$ и энергии электронного облучения $E_{0}=5$ и $15 \mathrm{keV}$. В случае облучения протонами с энергией $150 \mathrm{keV}$ резкого скачка потенциала в начальный момент времени не наблюдается. Вместо этого потенциал поверхности начинает расти плавно, начиная с первых секунд облучения электронами. Для энергии электронного зонда $E_{0}=15 \mathrm{keV}$ (рис. $3, b$ ) картина зарядки кварца после протонного облучения в целом похожа, за исключением случая предварительного облучения протонами с энергий $E_{\mathrm{H}^{+}}=50 \mathrm{keV}$. В этом случае равновесный поверхностный потенциал, достигаемый при облучении электронами с меньшей плотностью тока, оказывается больше, чем при облучении с большей плотностью тока. Из рис. 2 следует, что зарядка облученного протонами сапфира начинается гораздо раньше, чем зарядка исходного, не облученного образца. Это говорит о наличии в облученном диэлектрике большого количества центров захвата электронов.

Для того чтобы понять различия в кинетике зарядки диэлектрических образцов в случае их предварительного облучения протонами и ионами, обратимся к рис. 4, на котором представлена схема зарядки для случая протонного $(a)$ и ионного $(b)$ облучения.

В первую очередь следует отметить, что энергии протонов (50 и $150 \mathrm{keV})$ значительно превышают энергии, используемые в экспериментах с ионными пучками $(10$ и $15 \mathrm{keV})$. Это приводит к тому, что глубина проникновения протонов в образцы значительно больше глубины проникновения ионов $(15-20 \mathrm{~nm})$. По расчетам, проведенным с помощью программы SRIM [10], глубины проникновения протонов с энергией $50 \mathrm{keV}$ составляют $660 \mathrm{~nm}$ для $\mathrm{Al}_{2} \mathrm{O}_{3}$ и $690 \mathrm{~nm}$ для $\mathrm{SiO}_{2}$, а протонов с энергией $150 \mathrm{keV}$ соответственно 1600 и $1750 \mathrm{~nm}$. Поэтому положительный заряд, привносимый в диэлектрический образец протонами $\mathrm{Q}_{2}^{+}$(рис. 4,a), аккумулируется на большей глубине по сравнению с ионным облучением $\mathrm{Ar}^{+}$и $\mathrm{Ga}^{+}$(рис. 4, $b$ ). Этот поло- жительный заряд создает внутреннее поле $\bar{E}_{2}$, направленное к поверхности, и значительно влияет на процесс последующей зарядки под электронным пучком.

Согласно двуслойной модели зарядки диэлектриков $[11,12]$, при электронной бомбардировке мишени вблизи ее поверхности образуется слой положительного заряда из-за эмиссии вторичных электронов. Толщина этого слоя $\lambda_{s e}$ определяется глубиной выхода вторичных электронов $(10-20 \mathrm{~nm})$ и оказывается чуть меньше слоя положительного заряда $Q^{+2}$, привносимого ионами $\mathrm{Ar}$ и $\mathrm{Ga}$. Одновременно на глубине $h$ образуется более протяженный слой отрицательного заряда $Q$ от аккумулированных на глубоких и мелких ловушках первичных электронов. Толщина этого слоя приближенно равна половине длины пробега первичных электронов $R_{0}$ (порядка долей и единиц $\mu \mathrm{m})$. Уравновешивающим фактором, ограничивающим рост положительных и отрицательных потенциалов до равновесных значений, является радиационно-индуцированный ток IRIC, возникающий между заряженными слоями под действием внутренних полей $\bar{E}_{1}$ и $\bar{E}_{2}$.

Таким образом, в случае предварительной имплантации протонов с энергией $150 \mathrm{keV}$ в диэлектрике при электронном облучении формируются три слоя заряда, создающие электрические поля $\bar{E}_{1}$ и $\bar{E}_{2}$, направленные в противоположные стороны. В случае предварительного облучения протонами с энергией $50 \mathrm{keV}$ слой положительного заряда формируется на глубине около $0.7 \mu \mathrm{m}$, соответствующей глубине пробега первичных электронов. В этом случае первичные электроны просто компенсируют ранее накопленный в диэлектрике положительный заряд.

\section{Заключение}

Установлено, что предварительное воздействие на диэлектрическую поверхность ионизирующего корпус- 
кулярного излучения (протонов и ионов) значительно изменяет зарядовые характеристики поверхности.

Экспериментальные исследования влияния предварительного облучения ионами на процесс электронной зарядки диэлектрика показали существенную разницу в кинетике зарядки после облучения ионами инертных газов, ионами металлов и протонами.

Возможна как усиленная электризация диэлектрической мишени ионами $\mathrm{Ar}^{+}$, так и ее более медленная зарядка ионами $\mathrm{H}^{+}$, что объясняется различными механизмами радиационно-стимулированного дефектообразования и различием в энергиях имплантируемых ионов в сравнении с энергиями облучающих электронов, определяющими глубину залегания положительно и отрицательного слоев зарядов соответственно.

Полученные результаты интерпретированы в рамках модели, учитывающей различные радиационностимулированные процессы дефектообразования и эффект десорбции кислорода на поверхности диэлектрика при его облучении электронами низких и средних энергий.

\section{Финансирование работы}

Работа выполнена в рамках проекта РФФИ 18-0200813A.

\section{Конфликт интересов}

Авторы заявляют, что у них нет конфликта интересов.

\section{Список литературы}

[1] Liebault J., Siesse-Moya D., Moya F., Zarbout K., Damamme G., Moya G. Annual Report - Conference on Electrical Insulation and Dielectric Phenomena. 2002. P. 652-655.

[2] Vance D.W. // Annual Report - Conference on Electrical Insulation and Dielectric Phenomena. 1970. P. 1-7.

[3] Рау Э.И., Татаринщев А.А., Зыкова Е.Ю., Иваненко И.П., Купреенко С.Ю., Миннебаев К.Ф., Хайдаров А.А. // ФТТ. 2017. T. 59. C. $1504-1509$.

[4] Rau E.I., Tatarintsev A.A., Khvostov V.V., Yurasova V.E. // Vacuum. 2016. Vol. 129. P. 142-148.

[5] Kröger F.A. Advances in Ceramics, Structure and Properties of $\mathrm{MgO}$ and $\mathrm{Al}_{2} \mathrm{O}_{3}$ Ceramics / Ed. by W.D. Kingery. Columbus: American Ceramic Society, 1984. Vol. 10.

[6] Alves E., da Silva M.F., Marques J.G., Soares J.C., Freitag K. // Nucl. Instrum. Meth. B. 1998. Vol. 141. P. 353-358.

[7] Alves E., Marques C., da Silva R.C., Monteiro T., McHargue C. // Surf. Coat. Technol. 2009. Vol. 203. P. 2357-2362.

[8] Privezentsev V., Goryachev A., Shcherbachev K. // Proc. of the World Congress on New Technologies (New Tech. 2015). Barcelona, Spain, 2015. P. 453-454.

[9] Yogev S., Levin J., Molotskii M., Schwarzman A., Avayu O., Rosenwaks Y. // J. Appl. Phys. 2008. Vol. 103. P. 064107.

[10] SRIM - The Stopping and Range of Ions in Matter. [Электронный ресурc] // URL: http://www.srim.org
[11] Cazaux J. // J. Appl. Phys. 2001. Vol. 89. P. 8265-8272.

[12] Melchinger A., Hofmann S. // J. Appl. Phys. 1995. Vol. 78. P. 6224-6231. 